Document downloaded from:

http://hdl.handle.net/10251/63411

This paper must be cited as:

Gómez Tejedor, JA.; Monsoriu Serra, JA. (2015). Characterizing the movement of a falling rigid rod. European Journal of Physics. 36. doi:10.1088/0143-0807/36/5/055036.

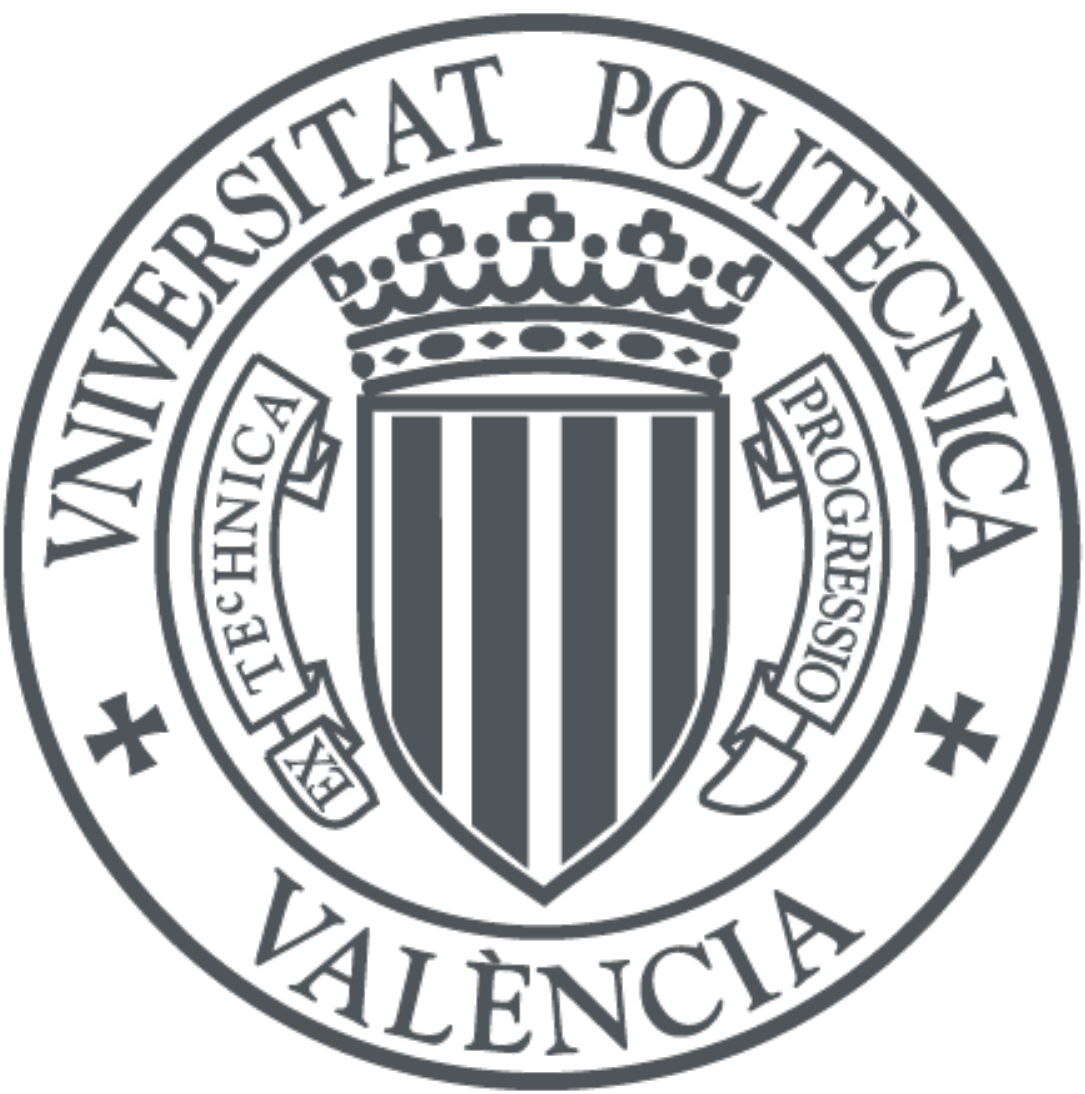

The final publication is available at

http://dx.doi.org/10.1088/0143-0807/36/5/055036

Copyright European Physical Society

Additional Information 


\title{
Characterising the movement of a falling rigid rod
}

\author{
José A. Gómez-Tejedor
}

Centre for Biomaterials and Tissue Engineering, CBIT, Universitat Politècnica de

València, Camí de Vera s/n, 46022, València, Spain.

E-mail: jogomez@fis.upv.es

\section{Juan A. Monsoriu}

Centro de Tecnologías Físicas, Universitat Politècnica de València, Camí de Vera s/n, 46022, València, Spain.

E-mail: jmonsori@fis.upv.es

January 2015

European Journal of Physics 36 2015: 055036

doi:10.1088/0143-0807/36/5/055036

\begin{abstract}
.
In this paper we present a simple experimental set-up to study the fall of a rigid rod, which can freely rotate around an articulated joint at the lowest point. The experimental set-up permits preparation of a laboratory session for Physics or Engineering students. The analysis of the data is oriented at several degrees of difficulty, in such a way that the same experimental set-up can be used with students on different courses. The experimental data obtained with an electro-optical sensor are fitted to the theoretical equation of motion, obtaining a very good agreement between experiment and theory. In addition, direct measurement of the parameters involved in the equations was carried out, showing a very good agreement with the calculated parameters.
\end{abstract}

PACS numbers: 07.10.-h, 45.20.-d, 45.20.dc, 45.20.dh, 45.40.Cc

Keywords: rigid rod fall, electro-optical sensor, physical pendulum.

Submitted to: Eur. J. Phys. 
JA Gómez-Tejedor, JA Monsoriu, European Journal of Physics 36 2015: 055036, doi:10.1088/0143-0807/36/5/055036

\section{Introduction}

This paper is focused on the experimental study of the fall of a rigid rod, which can freely rotate around an articulated joint at the lowest point, whose fundamental equations are the same as for a pendulum. Analytical and approximate solutions for the differential equation of the pendulum can be found in the literature (see for instance references [1, 2, 3, 4, 5, 6, 7] among many others, and references therein). These solutions are based on Jacobi elliptic functions 8 .

For the experimental study of a falling rigid rod, a home-made experimental setup is presented, which consists of a rigid rod free to rotate in a vertical plane. This experimental set-up is shown in Figure1. The set-up permits preparation of a laboratory session for Physics or Engineering students. The analysis of the data is oriented at several degrees of difficulty, in such a way that the same experimental set-up can be useful for students on different courses. In addition, the cost of the proposed system is very cheap, except for the personal computer, which is used for data recording, although this should not be an implementation difficulty as this is a very common general equipment in Physics laboratories. Experimental measurements can also be done by using a high speed camera, as reported in [9], where the movement of a rigid rod is compared to the free fall of a ball, to find which hits earlier the ground, which is commonly described as faster than gravity, faster than g, free fall paradox or simply falling stick experiment.

The experimental set-up shown in Figure 1(a) consists of two rigid rods with an articulated joint at the lowest point. These rigid rods can fall around the articulated joint. At the end of the rod, two pieces of a sponge are placed to cushion the fall, and to stop the system without being damaged (marked by a red circle in Figure 1a). Between the rigid rods there is a wooden half circle protractor marked in degrees, with 18 holes, together with an electro-optical sensor for detecting light passing through the holes. The holes have an angular size of 2 degrees. The first hole is placed at 7 degrees due to the size of the detection system. The electro-optical sensor (see Figure 1(b)) is composed of a light-emitting diode (LED) and a receiver phototransistor placed one in front of the other. When the system passes in front of the holes, the radiated light from the LED is received by the phototransistor producing a current which can be measured. The wire of the electro-optical sensor has to be placed very carefully to avoid influencing the mechanical motion of the system.

The system is connected to the mic-input of a sound-card [10] with a cable which has a capacitor in series, so a signal is detected when it passes in front of the hole edges, i.e. each 2 degrees, thus data about angular position vs. time can be measured (see Figure 2 and Table 1 in the results section). 

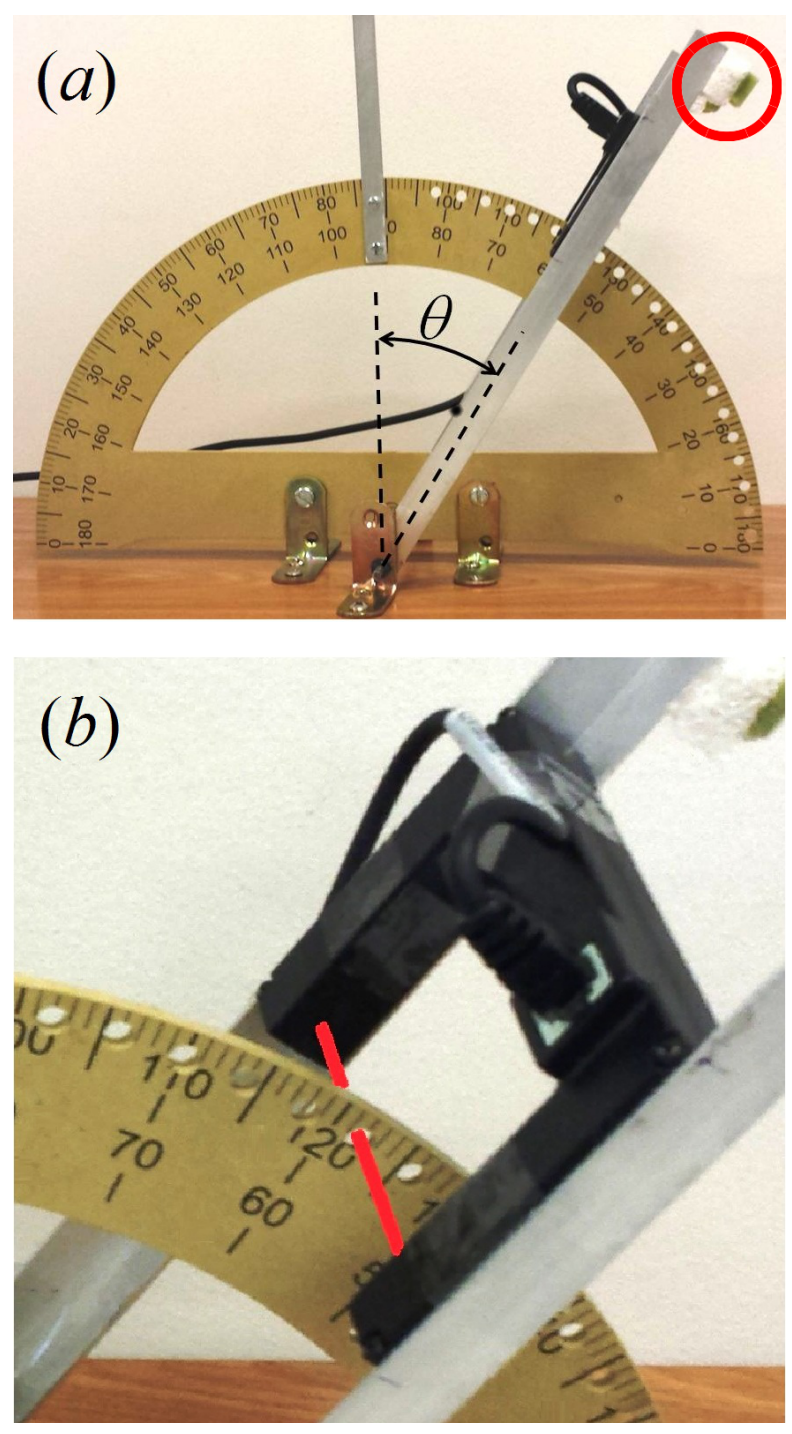

Figure 1. (a) Experimental set-up for measuring the free fall of a rigid rod. (b) Electro-optical sensor for detecting light passing through the holes. The red line represents the emitted light from the diode integrated in the sensor.

\section{Physical description of a falling rigid rod}

The problem is schematically represented in Figure 1(a), where $\theta$ is the angle between the vertical line and the rod. As commented in the introduction, the mathematical and physical description of the problem can be analyzed in several ways in order to adjust the problem to different student levels. In all cases, the assumption is made that damping is small enough to be neglected from the equations and subsequent analysis. This assumption will be discuss in section 4 .

The potential energy of the system when the rod makes an angle $\theta$ with the vertical line is given by

$$
U=m g h=m g R_{C M} \cos \theta,
$$


where $m$ is the total mass of the system, $g$ is the gravitational acceleration, $h$ is the height of the center of mass and $R_{C M}$ is the distance from the articulated joint to the center of mass of the system. The kinetic energy is given by

$$
K=\frac{1}{2} I \dot{\theta}^{2}=\frac{1}{2} m R_{g}^{2} \dot{\theta}^{2},
$$

where $\dot{\theta}$ is the angular velocity, $I$ is the moment of inertia, and $R_{g}$ is the radius of gyration defined as

$$
R_{g}=\sqrt{\frac{I}{m}} .
$$

The system is dropped from an initial angle $\theta_{0}$, without initial velocity. Then, the total initial energy, $E_{i}$, is given only by the potential energy at that position

$$
E_{i}=m g R_{C M} \cos \theta_{0} .
$$

From the energy conservation of mechanical energy, this initial energy should be equal to the total energy when the rod makes an angle $\theta$ with the vertical line,

$$
m g R_{C M} \cos \theta_{0}=m g R_{C M} \cos \theta+\frac{1}{2} m R_{g}^{2} \dot{\theta}^{2} .
$$

Therefore, we can obtain the angular velocity as

$$
\dot{\theta}=\sqrt{\frac{2 g R_{C M}}{R_{g}^{2}} \cos \theta_{0}-\frac{2 g R_{C M}}{R_{g}^{2}} \cos \theta} .
$$

Defining the constant $\Omega_{0}^{2}$ as

$$
\Omega_{0}^{2}=\frac{g R_{C M}}{R_{g}^{2}},
$$

Equation 6 can be reduced to

$$
\dot{\theta}=\Omega_{0} \sqrt{2\left(\cos \theta_{0}-\cos \theta\right)} .
$$

\section{Results}

In this section, the experimental results obtained using the described experimental setup are summarized, together with the parameter calculations. All calculations are carried out with Wolfram Mathematica ${ }^{\circledR}$ software version 9.0.1.0.

The square of Equation 8 is given by

$$
\dot{\theta}^{2}=\left(-2 \Omega_{0}^{2}\right) \cos \theta+\left(2 \Omega_{0}^{2}\right) \cos \theta_{0} .
$$

Using this expression, students should measure the angular velocity as a function of the angular position. The angular velocity can be approximately calculated as the size of the hole ( 2 degrees $=0.0349 \mathrm{rad}$ in the proposed set-up) divided by the time it 
takes to pass over the hole, which is measured with Audacity ${ }^{\circledR}$ software version 2.0 .5 [11]

$$
\dot{\theta} \approx \frac{\Delta \theta}{\Delta t}
$$

Within this approximation, students should graphically represent the square of the angular velocity vs. the cosine of the angle position, and a straight line should be obtained. From this data, performing a linear fit, the slope of the straight line is equal to $-2 \Omega_{0}^{2}$. The y-intercept of the line is equal to $2 \Omega_{0}^{2} \cos \theta_{0}$, subsequently the value of the initial angle can be calculated. This method is the simplest one of all those proposed, therefore it should be appropriate for first year students of Physics or Engineering degrees.

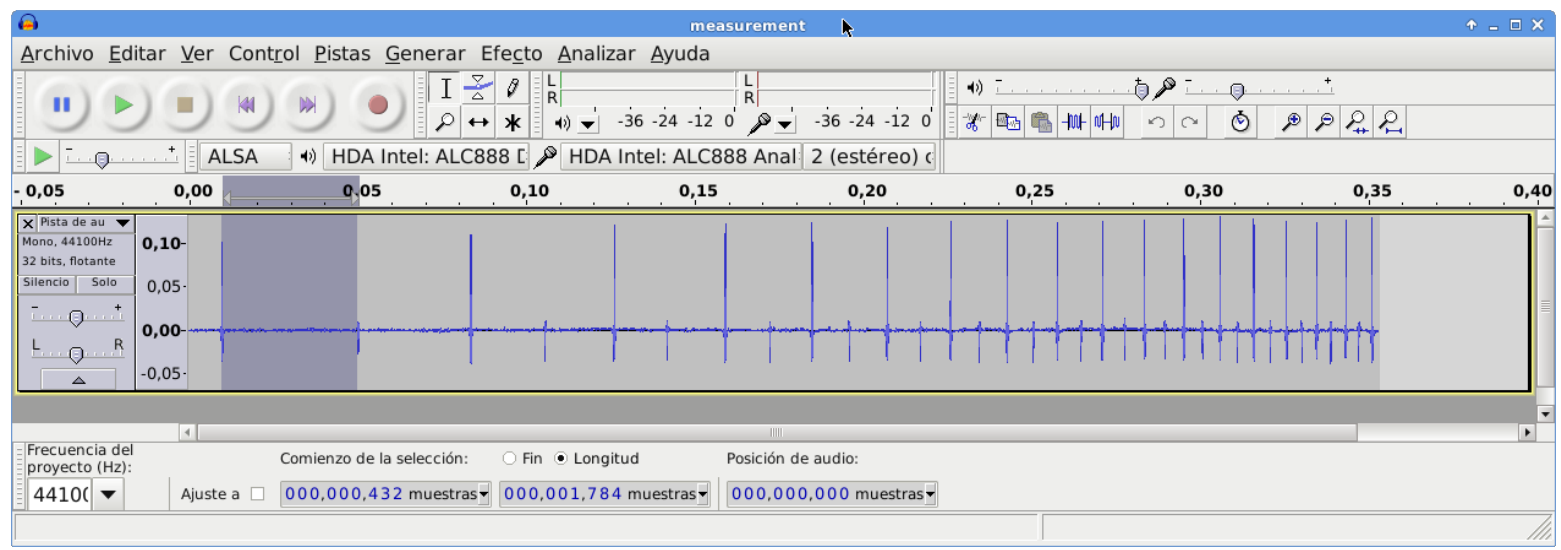

Figure 2. Example of a measurement with the Audacity ${ }^{\circledR}$ software.

In Figure 2 a picture of the computer screen is shown, where the experimental results can be observed. When the system detector passes in front of the edges of the hole, a signal is recorded by the Audacity software. Then, positioning the cursor at the first signal point (this point is taken as $t=0$ ) and the second cursor at the following points, the time can be measured. For instance, in Figure 2, the first point is measured, with a time interval between the first point and the second one of 1784 samples. Taking into account that the system measures at a rate of 44100 samples per second, we obtain the value of $\Delta t=1784 / 44100=40.5 \mathrm{~ms}$. In order to make accurate measurements, the time scale in the Audacity software has to be amplified. The complete set of data is shown in Table 1, where the angle $\theta$ is the angle between the rod and the vertical line. For the measurement of the angle, an error of 0.5 degrees is estimated. For time, the soundcard records data at a rate of 44100 points per second. However, considering an error of $1 / 44100 \mathrm{~s}$ is completely unrealistic, therefore an error of $1.4 \mathrm{~ms}$ is estimated, which is the minimum time that can be accurately measured using the Audacity software.

From the data shown in Table 1 and using Equation 10 the angular velocity can be calculated. In Figure 3, the square of the angular velocity as a function of angular position is represented. A linear fit has also been carried out in order to obtain the slope 
JA Gómez-Tejedor, JA Monsoriu, European Journal of Physics 36 2015: 055036, doi:10.1088/0143-0807/36/5/055036

Table 1. Experimental data measured with Audacity software.

\begin{tabular}{ll||ll}
\hline $\begin{array}{l}\theta \pm \mathbf{0 . 5}) \\
\text { (degrees) }\end{array}$ & $\begin{array}{l}(t \pm \mathbf{1 . 4}) \\
(\mathbf{m s})\end{array}$ & $\begin{array}{l}(\theta \pm \mathbf{0 . 5}) \\
(\text { degrees })\end{array}$ & $\begin{array}{l}(t \pm \mathbf{1 . 4}) \\
(\mathbf{m s})\end{array}$ \\
\hline 7.0 & 0.0 & 9.0 & 40.5 \\
11.0 & 73.9 & 13.0 & 95.9 \\
15.0 & 116.4 & 17.0 & 132.1 \\
19.0 & 149.3 & 21.0 & 162.5 \\
23.0 & 175.0 & 25.0 & 186.0 \\
27.0 & 197.4 & 29.0 & 207.2 \\
31.0 & 216.1 & 33.0 & 224.7 \\
35.0 & 232.9 & 37.0 & 240.7 \\
39.0 & 247.9 & 41.0 & 254.9 \\
43.0 & 261.2 & 45.0 & 267.8 \\
47.0 & 273.7 & 49.0 & 279.7 \\
51.0 & 285.4 & 53.0 & 290.9 \\
55.0 & 296.1 & 57.0 & 301.3 \\
59.0 & 306.1 & 61.0 & 311.0 \\
63.0 & 315.8 & 65.0 & 320.4 \\
67.0 & 324.8 & 69.0 & 328.9 \\
71.0 & 333.2 & 73.0 & 337.2 \\
75.0 & 341.2 & & \\
\hline
\end{tabular}

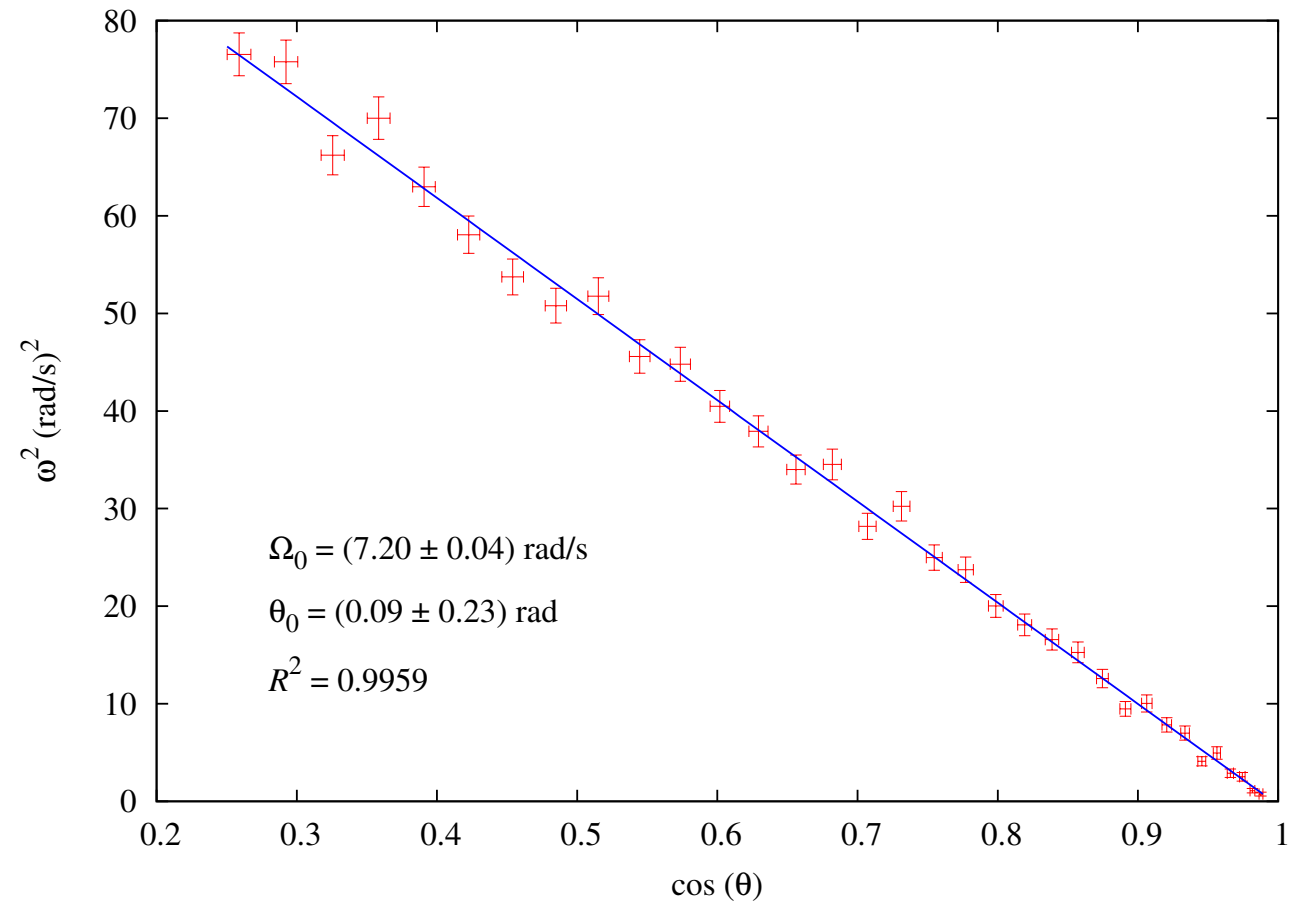

Figure 3. Angular velocity squared vs. $\cos \theta$. Crosses represent data points with error bars, and solid lines the linear fit. 
JA Gómez-Tejedor, JA Monsoriu, European Journal of Physics 36 2015: 055036,

doi:10.1088/0143-0807/36/5/055036

and the y-intercept point, comparison with Equation 9 , gives $2 \Omega_{0}^{2}=(103.7 \pm 1.2)(\mathrm{rad} / \mathrm{s})^{2}$ and $2 \Omega_{0}^{2} \cos \theta_{0}=(103.3 \pm 0.9)(\mathrm{rad} / \mathrm{s})^{2}$. From these results, the values of $\Omega_{0}$ and $\theta_{0}$ shown in Figure 3 are obtained.

\subsection{Analytical solution for a falling rigid rod}

Returning to Equation 8, and taking into account that the angular velocity is equal to the derivative of the angular position

$$
\frac{d \theta}{d t}=\Omega_{0} \sqrt{2\left(\cos \theta_{0}-\cos \theta\right)},
$$

a separation of the variables obtains

$$
d t=\frac{d \theta}{\Omega_{0} \sqrt{2\left(\cos \theta_{0}-\cos \theta\right)}} .
$$

The integration of this equation from $t=t_{0}$ where the rod makes an angle of $\theta_{0}$ with the vertical line, to a time $t$ where the angle is equal to $\theta$ leads to

$$
\begin{gathered}
t-t_{0}=\frac{1}{\Omega_{0} \sqrt{2}} \int_{\theta_{0}}^{\theta(t)} \frac{d \theta}{\sqrt{\cos \theta_{0}-\cos \theta}}, \\
t=t_{0}+\frac{\sqrt{2}}{\Omega_{0} \sqrt{\cos \left(\theta_{0}\right)-1}}\left(F\left(\frac{\theta(t)}{2}, \csc \left(\frac{\theta_{0}}{2}\right)\right)-F\left(\frac{\theta_{0}}{2}, \csc \left(\frac{\theta_{0}}{2}\right)\right)\right),
\end{gathered}
$$

where $F(\varphi, k)$ is the elliptic integral of the first kind, defined as follows [2, 3, 8]:

$$
F(\varphi, k)=\int_{0}^{\varphi} \frac{d \theta}{\sqrt{1-k^{2} \sin ^{2} \theta}}
$$

where $\varphi$ is the amplitude and $k$ is the parameter. Equation 15 is real for arbitrary value of $\varphi$ if $k^{2}<1$ and for $|\varphi|<\sin ^{-1}(1 / k)$ if $k^{2} \geq 1$. In this case, $k^{2}=\csc ^{2}\left(\frac{\theta_{0}}{2}\right)=$ $1 / \sin ^{2}\left(\frac{\theta_{0}}{2}\right) \geq 1$ and $\sin ^{-1}(1 / k)=\theta_{0} / 2$, and therefore the elliptic integrals of Equation 14 are imaginary for $\theta(t)>\theta_{0}$. Nevertheless, the final result of Equation 14 is always real for $\theta(t)>\theta_{0}$. Equation 14 gives the time $t=t\left(\theta_{i}, t_{0}, \Omega_{0}, \theta_{0}\right)$ as a function of the angle $\theta$, where $t_{0}, \Omega_{0}$ and $\theta_{0}$ are parameters which depend on the initial conditions.

In order to obtain these parameters, the method of least squares is used[12]. In the least squares fitting method the objective function is defined by

$$
\chi^{2}=\sum_{i=1}^{N} \frac{\left(t_{i}-t\left(\theta_{i}, t_{0}, \Omega_{0}, \theta_{0}\right)\right)^{2}}{\Delta t_{i}^{2}},
$$

where $N$ is de number of data points, $t\left(\theta, t_{0}, \Omega_{0}, \theta_{0}\right)$ is the fit function given by Equation 14, and $\Delta t_{i}$ is the error in the measurement of time, which in this case is constant for all data points. Minimization of this function over the fit parameters $t_{0}, \Omega_{0}$ and $\theta_{0}$ yields the least squares solution. This method is more complicated than the previous one, therefore it is recommended for advanced undergraduate students of Physics or Engineering degrees. 
JA Gómez-Tejedor, JA Monsoriu, European Journal of Physics 36 2015: 055036, doi:10.1088/0143-0807/36/5/055036

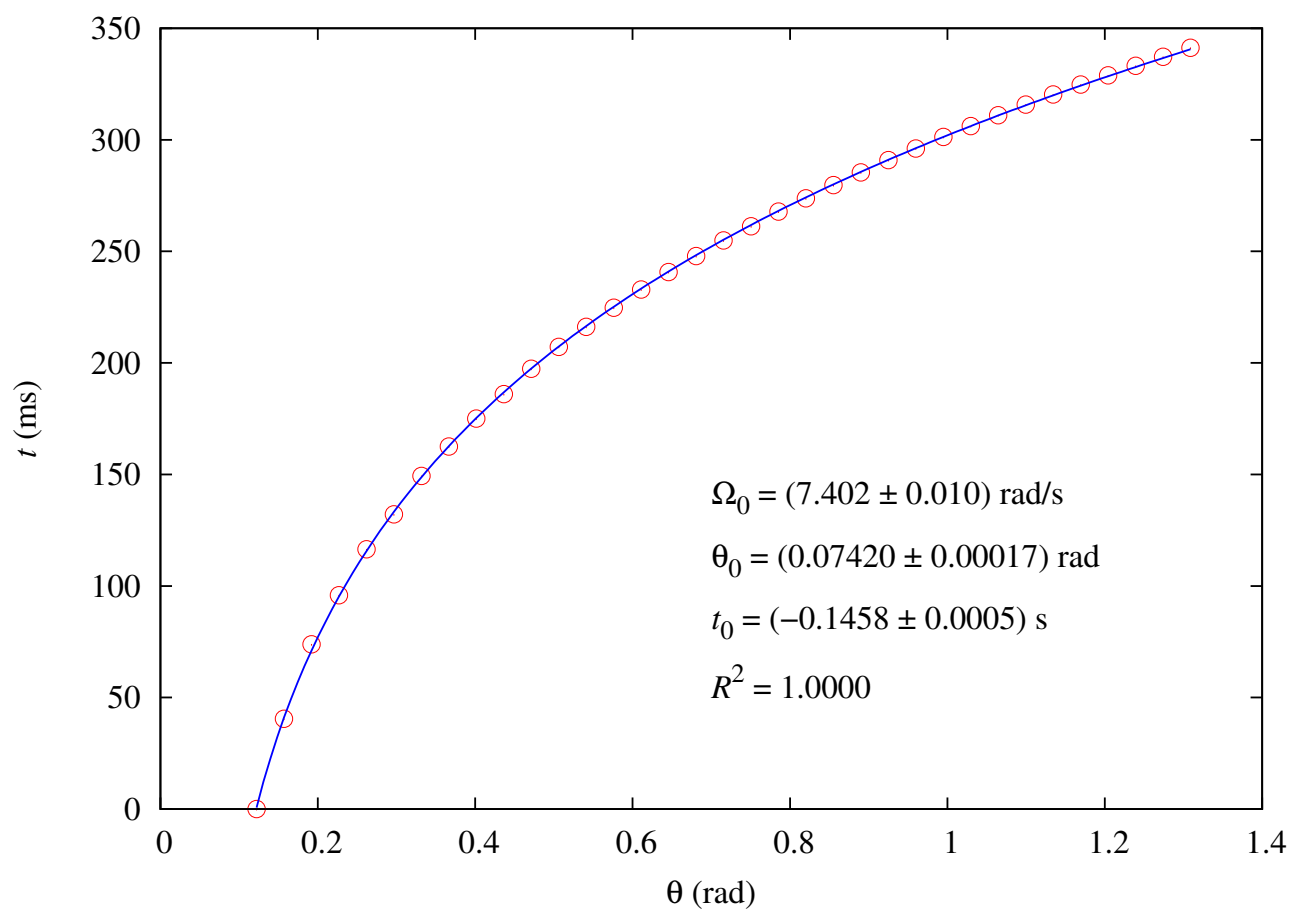

Figure 4. Time as a function of position angle. Dots represent data points and the solid line the analytical solution.

In Figure 4, time $t$ as a function of the angle $\theta$ is represented, comparing the experimental data with the analytical solution of time as a function of the angle (Equation 14). By using the method of least squares, the parameters $t_{0}, \Omega_{0}$ and $\theta_{0}$ are obtained. The results are shown in the Figure.

\subsection{Numerical solution of the differential equation of a falling rigid rod}

The equation of motion for a falling rigid rod is found by use of the Lagrangian function $L\left(q_{i}, \dot{q}_{i}\right)=K\left(q_{i}, \dot{q}_{i}\right)-U\left(q_{i}\right)$, where $q_{i}, \dot{q}_{i}$ are the generalized coordinates and velocities, and $U$ and $K$ the potential and kinetic energies introduced in Equations 1 and 2 . The differential equation of motion is given by Lagrange's equation

$$
\frac{d}{d t} \frac{\partial L\left(q_{i}, \dot{q}_{i}\right)}{\partial \dot{q}_{i}}-\frac{\partial L\left(q_{i}, \dot{q}_{i}\right)}{\partial q_{i}}=0 .
$$

This problem has only one degree of freedom, $q_{i}=\theta$. Therefore, Lagrange's Equation 17 applied to Equations 1 and 2 yields the differential equation for $\theta$

$$
\frac{d^{2} \theta}{d t^{2}}-\frac{g R_{C M}}{R_{g}^{2}} \sin \theta=0,
$$

and introducing the constant $\Omega_{0}$ defined in Equation 7

$$
\frac{d^{2} \theta}{d t^{2}}-\Omega_{0}^{2} \sin \theta=0
$$


which is the well known equation of the movement of a pendulum [13, 14, 15, 16], but in this case in upward position. This equation could also be obtained by relating the torque with the variation of the angular momentum with time, as it is done in reference [9].

This differential equation can be solved analytically in a closed form with exact solutions in terms of Jacobian Elliptic Functions [2, 17]. However, in this section, we are interested in finding a numerical solution of this differential equation, with the initial conditions for $t=t_{0}$

$$
\theta\left(t=t_{0}\right)=\theta_{0}, \quad \dot{\theta}\left(t=t_{0}\right)=0 .
$$

In this case, the free parameters $t_{0}, \Omega_{0}$ and $\theta_{0}$ have to be fitted again. Therefore, once more the least squared method is used to obtain these free parameters. In this case, the objective function is defined as

$$
\chi^{2}=\sum_{i=1}^{N} \frac{\left(\theta_{i}-\theta_{N S}\left(t_{i}, t_{0}, \Omega_{0}, \theta_{0}\right)\right)^{2}}{\Delta \theta_{i}^{2}},
$$

where $\Delta \theta_{i}^{2}$ is the measurement error of the angle $\theta$ which is also constant. $\theta_{N S}\left(t, t_{0}, \Omega_{0}, \theta_{0}\right)$ represents the numerical solution for $\theta$ of the differential Equation 19. which depends on time $t$ and the parameters $t_{0}, \Omega_{0}$ and $\theta_{0}$. This method is also more complicated than the first one, therefore it is also recommended for advanced undergraduate students of Physics or Engineering degrees. Simplified numerical solutions are already possible at undergraduate level by using spreadsheet programs, as it is done in reference 9 .

In Figure 5, the angle $\theta$ is represented as a function of time $t$, and the numerical solution of the differential equation of the movement is compared (Equation 19p). The results for parameters $t_{0}, \Omega_{0}$ and $\theta_{0}$ are shown in the Figure.

\subsection{Direct measurement and small oscillations}

In order to compare the results obtained with the previously described methods, direct measurement of the parameters can be performed by the students. For the angle $\theta_{0}$, the initial position of the rod can be directly measured with the half circle protractor.

Equation 19 for small oscillations $(\sin \theta \approx \theta$ ) gives us the well known equation for simple harmonic motion

$$
\frac{d^{2} \theta}{d t^{2}}-\Omega_{0}^{2} \theta=0,
$$

where the $\Omega_{0}$ constant is related to the oscillation period, $T$, by $\Omega_{0}=2 \pi / T$. This equation of motion is the same for the pendulum in upward position as for the normal position except by a sign, and therefore we can assume that this constant $\Omega_{0}$ should be equal to the one calculated previously. Students can place the moving part of the rigid rod in the normal pendulum position, and make it oscillates over its equilibrium position. From the oscillation period, they can find an approximate value of the $\Omega_{0}$ 
JA Gómez-Tejedor, JA Monsoriu, European Journal of Physics 36 2015: 055036, doi:10.1088/0143-0807/36/5/055036

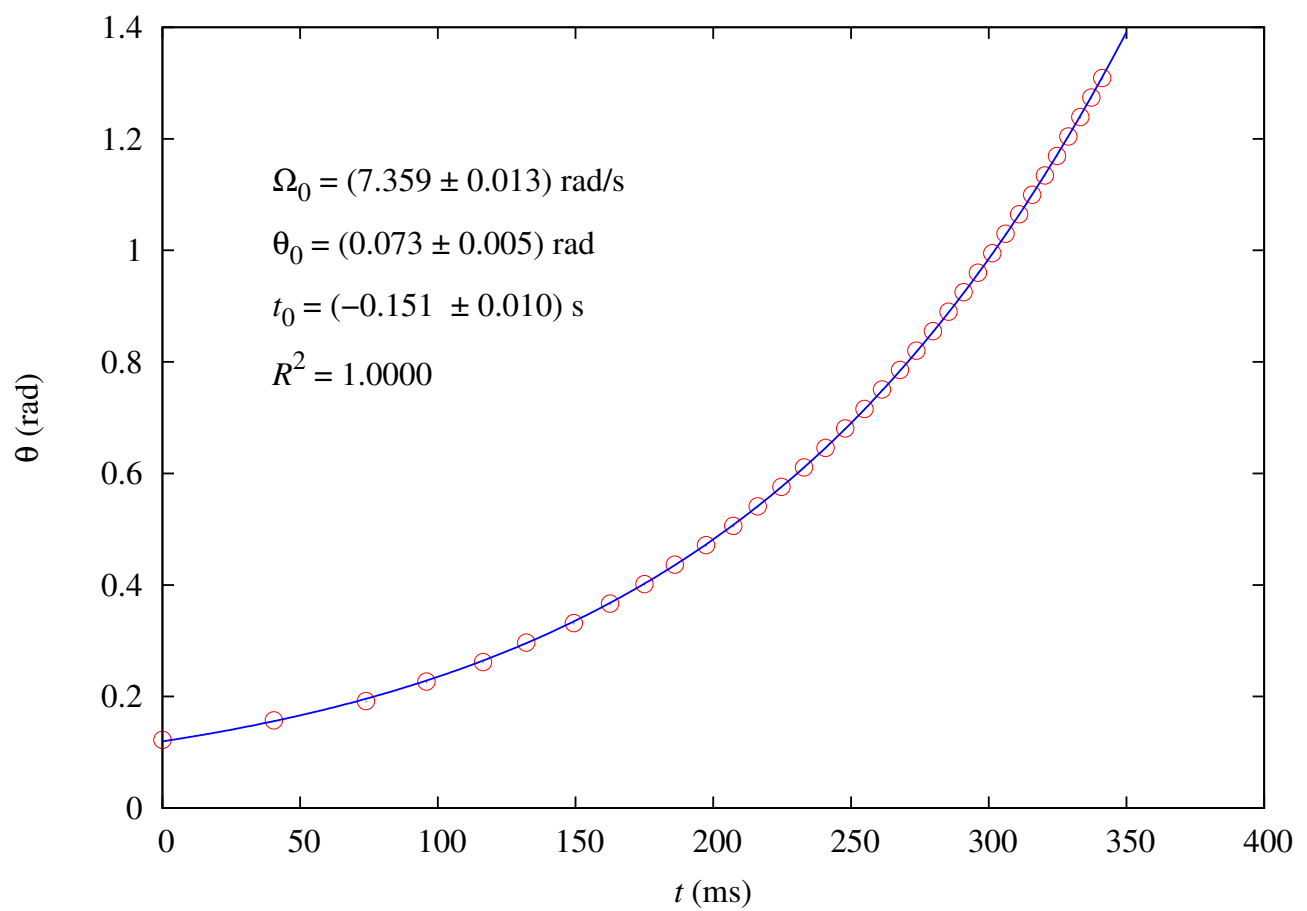

Figure 5. Position angle as a function of time. Dots represent data points, and the solid line the numerical solution of the differential equation.

constant. This approximation can be use for small oscillations. For instance for an initial oscillation amplitude of $20^{\circ}$, the discrepancy in the calculated period by this way is approximately equal to $1 \%[2]$.

\section{Discussion}

The results obtained for the value of the parameters are very similar in all four methods (see Table 2). Only the results of method A and D are slightly different compared to the other two. The small differences obtained with method A can be attributed to the approximation for the value of the angular velocity using Equation 10, which could introduce some errors, and for this reason, some points in the graph vary slightly from the trend line.

Table 2. Results obtained for the parameters using the three methods described in the text, and direct measurement.

\begin{tabular}{lllll}
\hline & $\Omega_{0}(\mathrm{rad} / \mathrm{s})$ & $\theta_{0}(\mathrm{rad})$ & $t_{0}(\mathrm{~s})$ & $R^{2}$ \\
\hline A. Angular velocity & $7.20 \pm 0.04$ & $0.09 \pm 0.23$ & & 0.9959 \\
B. Analytical solution & $7.402 \pm 0.010$ & $0.07420 \pm 0.00017$ & $-0.1458 \pm 0.0005$ & 1.0000 \\
C. Numerical solution & $7.359 \pm 0.013$ & $0.073 \pm 0.005$ & $-0.151 \pm 0.010$ & 1.0000 \\
D. Direct measurement & $7.09 \pm 0.06$ & $0.096 \pm 0.009$ & & \\
\hline
\end{tabular}

Comparing methods $\mathrm{B}$ and $\mathrm{C}$, it should be mentioned that the results for method 
$\mathrm{B}$ are slightly better than method $\mathrm{C}$, which can be seen in Table 2 in the value of the error of the parameters, which are smaller than method C. Also, it should be mentioned that the value of the coefficient of determination $R^{2}$ is closer to 1 for method $\mathrm{B}\left(R^{2}=0.99999999994\right)$ than for method C $\left(R^{2}=0.99997\right)$. In Table 2 five significant figures are given for $R^{2}$, and therefore this difference cannot be appreciated. This difference between both methods can be attributed to the numerical errors that could appear in the numerical solution of the differential equation in method $\mathrm{C}$.

The initial angle $\theta_{0}$ was also measured directly on the half circle protractor, obtaining the value $\theta_{0}=(0.096 \pm 0.009) \mathrm{rad}$. The agreement of this result with the results from previous methods is very good, especially for method A, although the error for this parameter in method $\mathrm{A}$ is very large. The reason of this large error for the angle $\theta_{0}$ in method $\mathrm{A}$ is due to the fact that in this method the value of $\cos \theta_{0}$, which is very close to 1 , has been calculated. When the arccos of this number is calculated and the propagation of error is performed, a large value for the error is obtained. For methods $\mathrm{B}$ and $\mathrm{C}$ a slightly smaller value for the angle $\theta_{0}$ than the direct measurement of the angle was found.

The value of $\Omega_{0}$ for the oscillation period was measured (method D), and a value of $\Omega_{0}=(7.09 \pm 0.06) \mathrm{rad} / \mathrm{s}$ was found under the small oscillations approximation. Again, method A provides a better approach for the direct measurement of $\Omega_{0}$, while methods $\mathrm{B}$ and $\mathrm{C}$ give a slightly greater value for the $\Omega_{0}$ constant. These small differences could be caused by the existence of a slight damping not considered in the oscillatory motion (Equation 22).

\section{Conclusions}

In this paper a home-made experimental set-up to measure the fall of a rigid rod, which can freely rotate around an articulated joint at the lowest point, is presented. This experimental set-up permits preparation of a laboratory session for Physics or Engineering students. The data analysis was carried out using three different methods.

The first method is the simplest for students, and can be performed by students in the first year of a Physics or Engineering degree. In this paper, all calculations are performed using Wolfram Mathematica, but the calculations needed to obtain the free parameters in the linear fit can be carried out with a spread-sheet or any linear fit software. In spite of the approximation realized to calculate the angular velocity, a very good agreement between the fitted parameters and direct measurement of these parameters was found.

The second and third methods are more complicated, and greater mathematical knowledge is needed to perform the required calculations. For this reason, the authors consider that these two methods are more appropriate for advanced Physics or Engineering students. Slight differences were found between the calculated parameters in the fit and the direct measurement.

In all cases a very good agreement between experiment data and theory was found, 
JA Gómez-Tejedor, JA Monsoriu, European Journal of Physics 36 2015: 055036, doi:10.1088/0143-0807/36/5/055036

with values for the coefficient of determination $R^{2}$ equal to 1 within five significant figures for methods $\mathrm{B}$ and $\mathrm{C}$, and very close to 1 for method $\mathrm{A}$.

\section{Acknowledgments}

Authors would like to thank the Institute of Education Sciences of the Universitat Politècnica de València (Spain) for support of the Teaching Innovation Groups MoMa and e-MACAFI and for the financial support through PIME Project PIME/2014/A/031/B. The translation of this paper was funded by the Universitat Politècnica de València, Spain.

\section{References}

[1] Fenn J G, Bayne D A and Sinclair B D 1998 American Journal of Physics 66 981-984 URL http://scitation.aip.org/content/aapt/journal/ajp/66/11/10.1119/1.19019

[2] Qureshi M I, Rafat M and Azad S I 2010 European Journal of Physics 31 1485 URL http: //stacks. iop.org/0143-0807/31/i=6/a=014

[3] Ochs K 2011 European Journal of Physics 32479 URL http://stacks.iop.org/0143-0807/32/ $i=2 / a=019$

[4] Beléndez A, Arribas E, Ortuño M, Gallego S, Márquez A and Pascual I 2012 Computers E Mathematics with Applications 641602 - 1611 ISSN 0898-1221 URL http://www. sciencedirect.com/science/article/pii/S089812211200017X

[5] Bel A, Reartes W and Torresi A 2012 European Journal of Physics 33231 URL http://stacks. iop.org $/ 0143-0807 / 33 / i=2 / a=231$

[6] Butikov E I 2012 European Journal of Physics 331555 URL http://stacks.iop.org/ $0143-0807 / 33 / i=6 / a=1555$

[7] Douvropoulos T G 2012 European Journal of Physics 33207 URL http://stacks.iop.org/ $0143-0807 / 33 / i=1 / a=207$

[8] Clark D N 2000 Dictionary of Analysis, Calculus, and Differential Equations Comprehensive Dictionary of Mathematics (CRC Press)

[9] Vollmer M and Möllmann K P 2012 European Journal of Physics 331277 URL http://stacks. iop.org $/ 0143-0807 / 33 / i=5 / a=1277$

[10] Abellán-García F J, García-Gamuz J A, Valerdi-Pérez R P and Ibáñez-Mengual J A 2012 European Journal of Physics 331271 URL http://stacks.iop.org/0143-0807/33/i=5/a=1271

[11] Mazzoni D and Dannenberg R 2014 Audacity http://audacity.sourceforge.net/ accessed: 2014-01-16

[12] Wolberg J 2005 Data Analysis Using the Method of Least Squares. Extracting the Most Information from Experiments. (Berlin: Springer-Verlag)

[13] Stephenson A 1908 Philosophical Magazine Series 615 233-236 URL http://www.tandfonline. com/doi/abs/10.1080/14786440809463763

[14] Phelps F M and Hunter J H 1965 American Journal of Physics 33 285-295 URL http: //scitation.aip.org/content/aapt/journal/ajp/33/4/10.1119/1.1971474

[15] Blitzer L 1965 American Journal of Physics 33 1076-1078 URL http://scitation.aip.org/ content/aapt/journal/ajp/33/12/10.1119/1.1971158

[16] Friedman M H, Campana J E, Kelner L, Seeliger E H and Yergey A L 1982 American Journal of Physics 50 924-931 URL http://scitation.aip.org/content/aapt/journal/ajp/50/10/ $10.1119 / 1.13015$

[17] Beléndez A, Pascual C, Méndez D, Beléndez T and Neipp C 2007 Revista Brasileira de Ensino de Física 29645 - 648 ISSN 1806 - 1117 URL http://sbfisica.org.br/rbef/pdf/070707.pdf 